\title{
Value of ultrasound combined with MRI in the diagnosis of primary and recurrent hepatocellular carcinoma
}

\author{
XIAOLI JI ${ }^{1 *}$, SHISHENG ZHOU ${ }^{2 *}$, PENG YANG ${ }^{3}$, FAQIN LIU ${ }^{4}, \mathrm{YAN} \mathrm{LI}^{4}$ and $\mathrm{HONG} \mathrm{LI}^{5}$ \\ ${ }^{1}$ Department of Special Inspection (Ultrasound in Obstetrics and Gynecology), \\ Qingdao Women and Children's Hospital, Qingdao, Shandong 266034; ${ }^{2}$ Department of Ultrasound, Yantaishan Hospital, \\ Yantai, Shandong 264000; ${ }^{3}$ Administrative Department (Outpatient) and ${ }^{4}$ Department of Operating Room, \\ The People's Hospital of Zhangqiu Area, Jinan, Shandong 250200; ${ }^{5}$ Department of Ultrasound, \\ Jining No. 1 People's Hospital, Jining, Shandong 272111, P.R. China
}

Received December 20, 2018; Accepted August 7, 2019

DOI: $10.3892 / \mathrm{ol} .2019 .10945$

\begin{abstract}
Ultrasound (US) combined with magnetic resonance imaging (MRI) in the diagnosis of primary hepatocellular carcinoma (PHCC) and recurrent hepatocellular carcinoma (RHCC) were compared. The clinical data of 329 patients with hepatocellular carcinoma (HCC) admitted to Qingdao Women and Children's Hospital from June 2015 to December 2017 were collected. One hundred and sixty patients with PHCC were regarded as the PHCC group, and the other 169 patients with RHCC were regarded as the RHCC group. US and MRI were used in the imaging diagnosis of both groups and the results of US combined with MRI, US, and MRI alone were compared. The lesion size in the PHCC group was significantly higher than that in the RHCC group $(\mathrm{P}<0.05)$. The MRI fast-in and fast-out rates of the two groups were significantly higher than those of the other three methods $(\mathrm{P}<0.05)$. The coincidence rate of MRI in the two groups was higher than that of computed tomography (CT), US, and US combined with MRI $(\mathrm{P}<0.05)$. The coincidence rates of CT, US, MRI, and US combined with MRI in PHCC group were significantly higher than those in RHCC group. In PHCC group, MRI was superior to the other methods in the detection of micro HCC $(\mathrm{P}<0.05)$. In RHCC group, MRI was significantly better than US in the detection of micro HCC $(\mathrm{P}<0.05)$. The sensitivity, specificity, positive predictive value and negative predictive value of MRI were significantly better than the other three methods $(\mathrm{P}<0.05)$. MRI alone has
\end{abstract}

Correspondence to: Dr Hong Li, Department of Ultrasound, Jining No. 1 People's Hospital, 6 Jiankang Road, Jining, Shandong 272111, P.R. China

E-mail: nqq5c8@163.com

*Contributed equally

Key words: primary hepatocellular carcinoma, magnetic resonance imaging, ultrasound, recurrent hepatocellular carcinoma, diagnostic value the best diagnostic efficacy for micro HCC-type lesions. The diagnostic efficacy of MRI, US, CT, and US combined with MRI in PHCC was better than those in RHCC. In addition to imaging examination, the diagnosis of RHCC should be combined with other indicators for comprehensive diagnosis.

\section{Introduction}

Primary hepatocellular carcinoma (PHCC), a tumor derived from hepatocytes or intrahepatic ductal epithelial cells, is one of the most common malignant tumors (1). Its mortality is second only to gastric and esophageal cancer, and PHCC ranks third among cancers of the digestive system. PHCC has clinical features of insidious onset, non-obvious early symptoms and rapid progression. Nevertheless, with the development of medical science, the technology of early diagnosis of PHCC has greatly improved, and more patients with PHCC can undergo radical treatment (2-4). However, studies have also reported that the cumulative incidence of recurrent hepatocellular carcinoma (RHCC) after PHCC is $70-88 \%$ (5), and the recurrence after PHCC is an important factor of survival prognosis (6).

Ultrasound (US) and magnetic resonance imaging (MRI) are used to determine the morphological characteristics of liver, judging by the size of the liver and the difference in signal and density between normal and diseased tissue $(7,8)$. US can determine the nature and condition of the lesion by observing the blood perfusion of a liver tumor. The characteristic indices of US diagnosis of hepatocellular carcinoma (HCC) is 'fast in and fast out', the arterial phase is rapidly enhanced, and the portal phase is rapidly declining and hypoechoic (9). However, it has been reported that some HCC does not meet the indices, especially RHCC, which may lead to missed diagnosis by US (10). 'Fast in and fast out' is also a characteristic indicator of MRI diagnosis of HCC. Moreover, MRI has a very high soft tissue resolution, which can display the anatomical image of the liver, and helps with the understanding of the function and pathology of the liver. The lesions detected by US can be characterized by MRI contrast-enhanced scans $(11,12)$.

Currently, the diagnosis of RHCC mainly relies on computed tomography (CT), US, MRI, and other influential 
examinations combined with past medical history. Usually, however, the selection of the imaging examination mainly follows the method used for the diagnosis of PHCC $(13,14)$. However, after undergoing various treatments, RHCC has more diverse imaging effects than those of $\mathrm{PHCC}$, and is more difficult to diagnose. The examination method of PHCC does not necessarily accurately and effectively detect the recurrent lesions $(15,16)$. Therefore, this study compared the diagnostic value of PHCC and RHCC by using US alone, MRI alone and US combined with MRI, in order to select a more appropriate and accurate influential examination method for PHCC, provide new imaging ideas for RHCC diagnostic screening, and improve the detection of lesions in RHCC and the prognosis of patients with PHCC.

\section{Patients and methods}

Research subjects. The clinical data of 329 patients with hepatocellular carcinoma (HCC) who were admitted to Qingdao Women and Children's Hospital (Qingdao, China) from June 2015 to December 2017 were collected. A total of 160 patients with PHCC were regarded as the PHCC group, with a total of 170 lesions, and 169 patients were regarded as the RHCC group, with a total of 195 lesions. Inclusion criteria: All HCC patients were diagnosed by pathology. Serum of the patients was taken before surgery, radiotherapy and chemotherapy. All RHCC patients with a medical history of HCC and complete clinical data were included, patients in the RHCC group who were referred to the HCC recurrence-type histopathological diagnosis were included, patients of $>18$ years of age, and patients who signed the informed consent form. Exclusion criteria: Patients with CT, US and MRI enhanced scan contraindications, patients with other basic organ diseases, such as severe heart, liver and kidney dysfunctions, pregnant women or patients who were breast-feeding, patients who have mental illness or abnormal brain conditions, patients with other malignant tumors, with continuous HCC, or with incomplete medical records. This study was approved by the Ethics Committee of Qingdao Women and Children's Hospital. The patients and their families were informed, and informed consents were signed. All patients who participated in this research had complete clinical data.

CT examination. MSCT scan examination was performed. A 256-slice spiral CT [Philips 128-row (256-layer) speed spiral CT scanner; Philips Healthcare] was used to scan the lungs of the patients. The current was set to $250 \mathrm{~mA}$, and the voltage to $120 \mathrm{kV}$. The thickness of the layer was $\sim 5 \mathrm{~mm}$, and a pitch was 1.0-1.5 $\mathrm{mm}$. The data and images scanned by CT were then processed by a computer, including three-dimensional, planar, surface or volume reconstruction, and CT enhancement scan was performed on a small number of patients.

US examination. iU22 Color Doppler US Diagnostic Instrument was used (Philips Healthcare). The transducer frequency was $3.5 \mathrm{~Hz}$. A routine US examination was performed to observe the location, size, number, shape, and blood supply of each lesion.

MRI examination. Conventional T1WI, T2WI, T2 fat suppression and diffusion-weighted imaging (DWI) were performed
Table I. HCC classification (according to lesion size).

Types

Diameter of lesions $(\mathrm{cm})$

\begin{tabular}{ll}
\hline Micro HCC & $\leq 2$ \\
Small HCC & $2-5$ \\
Large HCC & $5-10$ \\
Huge HCC & $>10$ \\
\hline
\end{tabular}

HCC, hepatocellular carcinoma.

using an Intera 1.5T MRI scanner (Philips Healthcare). Conventional MRI parameter settings: Layer thickness, $7.0 \mathrm{~mm}$; pitch, $5.0 \mathrm{~mm}$; matrix, 125x256; field of view, $35 \mathrm{~cm}$. DWI parameters: repetition time (TR), $1,500 \mathrm{msec}$; echo time (TE), $51.6 \mathrm{msec}$; b-value, $0.800 \mathrm{sec} / \mathrm{mm}^{2}$. Then, the sputum glucosamine (National Pharmacopoeia H10950272; Guangzhou Kangchen Pharmaceutical Co., Ltd.) was used as a contrast agent for enhanced scanning: Intravenous anterior venous high-pressure injection of $25 \mathrm{ml}$ of contrast agent, $20 \mathrm{sec}$ scan of the arterial phase after injection, $60 \mathrm{sec}$ scan portal vein period with 5 min delay period of scanning.

HCC imaging diagnostic indices. HCC used 'fast in and fast out' as a diagnostic imaging index, in the arterial phase during angiography, the echo/signal intensity and imaging results of the portal phase, defined as follows: Enhanced arterial phase, the hypoechoic/signal of portal phase is fast in and fast out; enhanced arterial phase, the isoechoic or hyperechoic/signal of portal phase is fast in and slow out.

Classification of HCC lesions. The classification criteria, according to the size of HCC lesions, are shown in Table I.

Statistical analysis. SPSS 19.0 statistical package (IBM Corp.) was used for statistical analysis. The measurement data were expressed as the mean $\pm \mathrm{SD}$ and analyzed by t-test. The enumeration data were expressed as $\mathrm{n}(\%)$ and Chi-square test was used for the comparison of the coincidence rates. ANOVA was used for comparison between multiple groups and the post hoc test was the Least Significant Difference test. The level of significance was set at $\alpha=0.05$.

\section{Results}

Comparison of general clinical data between the two groups. There were no differences in age, sex, HCC type, lesion location, total number of lesions and serum $\alpha$-fetoprotein (AFP) between the two groups $(\mathrm{P}>0.05)$. However, the lesion size in the PHCC group was significantly higher than that in the RHCC group $(\mathrm{P}<0.05)$, as shown in Table II.

Image performance after angiography in PHCC patients. In the PHCC group, there were 110 cases $(68.75 \%)$ of CT, 149 cases $(93.13 \%)$ of MRI, 121 cases $(75.63 \%)$ of US and 115 cases $(71.88 \%)$ of US combined with MRI, showing fast in and fast out. The fast in and fast out rate of MRI was significantly higher than those of the other three groups $(\mathrm{P}<0.05)$, 
Table II. General clinical data of the two groups of patients.

\begin{tabular}{|c|c|c|c|c|}
\hline Factors & PHCC group $(n=160)$ & RHCC group (n=169) & $\chi^{2} / \mathrm{t}$ value & $\mathrm{P}$-value \\
\hline Age (years) & $53.54 \pm 21.45$ & $55.76 \pm 23.54$ & 0.895 & 0.372 \\
\hline $\operatorname{Sex}[\mathrm{n}(\%)]$ & & & 3.011 & 0.083 \\
\hline Male & $120(75.00)$ & $112(66.27)$ & & \\
\hline Female & $40(25.00)$ & $57(33.73)$ & & \\
\hline HCC type $[\mathrm{n}(\%)]$ & & & 0.966 & 0.326 \\
\hline Bureau & $88(55.00)$ & $102(60.36)$ & & \\
\hline Nodular & $72(45.00)$ & $67(39.64)$ & & \\
\hline Lesion location $[\mathrm{n}(\%)]$ & & & 0.043 & 0.979 \\
\hline Left liver & $34(21.25)$ & $37(21.89)$ & & \\
\hline Right liver & $108(67.50)$ & $114(67.46)$ & & \\
\hline Left and right liver & $18(11.25)$ & $18(10.65)$ & & \\
\hline Lesion size $(\mathrm{cm})$ & $3.98 \pm 1.67$ & $3.23 \pm 1.32$ & 4.503 & $<0.001$ \\
\hline Total no. of lesions & $1.22 \pm 1.01$ & $1.06 \pm 0.96$ & 1.471 & 0.143 \\
\hline Serum AFP [n (\%)] & & & 0.275 & 0.600 \\
\hline$\geq 400 \mathrm{ng} / \mathrm{ml}$ & $126(78.75)$ & $137(81.07)$ & & \\
\hline$<400 \mathrm{ng} / \mathrm{ml}$ & $34(21.25)$ & $32(18.93)$ & & \\
\hline
\end{tabular}

PHCC, primary hepatocellular carcinoma; RHCC, recurrent hepatocellular carcinoma; HCC, hepatocellular carcinoma; AFP, $\alpha$-fetoprotein.

Table III. Image performance after angiography in PHCC patients $[\mathrm{n}(\%)]$.

\begin{tabular}{lcc}
\hline Methods & $\begin{array}{c}\text { Fast in and } \\
\text { fast out }\end{array}$ & $\begin{array}{c}\text { Fast in and } \\
\text { slow out }\end{array}$ \\
\hline CT & $110(68.75)^{\mathrm{a}}$ & $50(31.25)^{\mathrm{a}}$ \\
US & $121(75.63)^{\mathrm{a}}$ & $39(24.37)^{\mathrm{a}}$ \\
MRI & $149(93.13)$ & $11(6.88)$ \\
US combined with MRI & $115(71.88)^{\mathrm{a}}$ & $45(28.12)^{\mathrm{a}}$ \\
\hline${ }^{\mathrm{a}}<0.05$, compared with MRI in the same group. PHCC, primary \\
hepatocellular carcinoma; CT, computed tomography; US, ultra- \\
sound; MRI, magnetic resonance imaging.
\end{tabular}

and there was no significant difference among the other three groups (Table III).

Image performance after angiography in RHCC patients. In the RHCC group, there were 88 cases $(52.07 \%)$ of CT, 121 cases $(71.60 \%)$ of MRI, 98 cases $(57.99 \%)$ of US, and 78 cases $(46.15 \%)$ of US combined with MRI, showing fast in and fast out. The fast in and fast out rate of MRI was significantly higher than those of the other three groups $(\mathrm{P}<0.05)$, and there was no significant difference among the other three groups (Table IV).

Comparison of coincidence rate. According to the characteristics of the contrast image, 'fast in and fast out' is the characteristic of positive diagnosis, and the coincidence rate $=$ (the number of cases that were positive for the
Table IV. Image performance after angiography in RHCC patients $[\mathrm{n}(\%)]$.

\begin{tabular}{lcc}
\hline Methods & $\begin{array}{c}\text { Fast in and } \\
\text { fast out }\end{array}$ & $\begin{array}{c}\text { Fast in and } \\
\text { slow out }\end{array}$ \\
\hline CT & $88(52.07)^{\mathrm{a}}$ & $81(47.93)^{\mathrm{a}}$ \\
US & $98(57.99)^{\mathrm{a}}$ & $71(42.01)^{\mathrm{a}}$ \\
MRI & $121(71.60)$ & $48(28.40)$ \\
US combined with MRI & $78(46.15)^{\mathrm{a}}$ & $91(53.85)^{\mathrm{a}}$ \\
\hline
\end{tabular}

${ }^{\mathrm{a}} \mathrm{P}<0.05$, compared with MRI in the same group. RHCC, recurrent hepatocellular carcinoma; CT, computed tomography; US, ultrasound; MRI, magnetic resonance imaging.

pathology)/(the total number of cases). The coincidence rate of MRI alone in the RHCC group was higher than those of CT, US, and US combined with MRI $(\mathrm{P}<0.05)$. The coincidence rate of MRI in PHCC group was significantly higher than those of the CT, US, and US combined with MRI $(\mathrm{P}<0.05)$. There was no significant difference in the coincidence rate of CT, US, and the US combined with MRI $(\mathrm{P}>0.05)$. The coincidence rates of CT, US, MRI, and US combined with $\mathrm{MRI}$ in the PHCC group were significantly higher than those in the RHCC group, as shown in Table V.

Comparison of the coincidence rate of $C T$, US and MRI in PHCC patients. A total of 170 lesions were found via pathological examination of PHCC patients, including 100 micro, 53 small, 15 large, and 2 huge HCC lesions. In the CT examination, there were 36 micro HCC lesions (36\%), 22 small HCC 
Table V. Comparison of coincidence rate between the two groups [n (\%)].

\begin{tabular}{lcrr}
\hline Methods & RHCC group $(\mathrm{n}=169)$ & PHCC group $(\mathrm{n}=160)$ & $\chi^{2}$ value \\
\hline CT & $88(52.07)^{\mathrm{a}}$ & $106(66.25)^{\mathrm{a}}$ & 0.830 \\
US & $98(57.99)^{\mathrm{a}}$ & $121(75.63)^{\mathrm{a}}$ & 11.49 \\
MRI & $121(71.60)$ & $149(93.13)$ & $<0.001$ \\
US combined with MRI & $78(46.15)^{\mathrm{a}}$ & $115(71.88)^{\mathrm{a}}$ & $<.88$ \\
\end{tabular}

${ }^{\mathrm{a}} \mathrm{P}<0.05$, compared with MRI in the same group. RHCC, recurrent hepatocellular carcinoma; PHCC, primary hepatocellular carcinoma; $\mathrm{CT}$, computed tomography; US, ultrasound; MRI, magnetic resonance imaging.

Table VI. Comparison of coincidence rate of CT, US and MRI in the group of patients with PHCC [n (\%)].

\begin{tabular}{lccccc}
\hline Types & No. of lesions & CT & US & MRI & US combined with MRI \\
\hline Micro HCC & 100 & $36(36)^{\mathrm{a}}$ & $40(40)^{\mathrm{a}}$ & $54(54)$ & $43(43)^{\mathrm{a}}$ \\
Small HCC & 53 & $22(41.51)$ & $26(49.06)$ & $29(54.72)$ & $35(66.04)$ \\
Large HCC & 15 & $15(100)$ & $15(100)$ & $15(100)$ & $15(100)$ \\
Huge HCC & 2 & $2(100)$ & $2(100)$ & $2(100)$ & $2(100)$ \\
\hline
\end{tabular}

${ }^{\mathrm{a} P}<0.05$, compared with MRI in the same group. PHCC, primary hepatocellular carcinoma; CT, computed tomography; US, ultrasound; MRI, magnetic resonance imaging; HCC, hepatocellular carcinoma.

Table VII. Comparison of coincidence rate of CT, US and MRI in the group of patients with RHCC [n (\%)].

\begin{tabular}{lccccc}
\hline Types & No. of lesions & CT & US & MRI & US combined with MRI \\
\hline Micro HCC & 150 & $53(35.33)^{\mathrm{a}}$ & $55(36.67)^{\mathrm{a}}$ & $78(52.00)$ & $65(43.33)$ \\
Small HCC & 38 & $17(44.74)$ & $18(47.37)$ & $19(50.00)$ & $26(68.42)$ \\
Large HCC & 7 & $7(100.00)$ & $7(100.00)$ & $7(100.00)$ & $7(100.00)$ \\
Huge HCC & 0 & 0 & 0 & 0 & 0
\end{tabular}

${ }^{a} \mathrm{P}<0.05$, compared with MRI in the same group. RHCC, recurrent hepatocellular carcinoma; CT, computed tomography; US, ultrasound; MRI, magnetic resonance imaging; HCC, hepatocellular carcinoma.

lesions (41.51\%), 15 large HCC lesions (100.00\%) and 2 huge HCC lesions (100.00\%). In the US examination, there were 40 micro HCC lesions (40\%), 26 small HCC lesions (49.06\%), 15 large HCC lesions $(100.00 \%)$ and 2 huge HCC lesions (100.00\%). In the MRI examination, there were 54 micro HCC lesions (54\%), 29 small HCC lesions (54.72\%), 15 large HCC lesions $(100.00 \%)$ and 2 huge HCC lesions (100.00\%). In the US combined with MRI examination, there were 43 micro HCC lesions (43\%), 35 small HCC lesions (66.02\%), 15 large HCC lesions (100.00\%) and 2 huge HCC lesions (100.00\%). Since CT diagnostic efficiency is lower than the other three methods, it was considered as the normal control. When the lesion type of PHCC patients was small HCC, there were no significant differences in the coincidence rate among US, MRI, and US combined with MRI $(\mathrm{P}>0.05)$. When the lesion was large HCC, there were no significant differences in the coincidence rate among US, MRI, and US combined MRI methods $(\mathrm{P}>0.05)$. When the lesions were micro HCC, the coincidence rate of MRI alone was significantly higher than that of US and US combined with MRI $(\mathrm{P}<0.05)$. There was no significant difference in the coincidence rate between US and US combined with MRI (P>0.05) (Table VI).

Comparison of the coincidence rate of CT, US and MRI in the RHCC patients. A total of 195 lesions were found via pathological examination of patients with RHCC. Among them, there were 150 lesions of micro HCC, 38 lesions of small HCC and 7 lesions of large HCC. Pathology, US, MRI, and US combined with MRI did not show any large HCC lesions. In the CT examination, there were 53 micro HCC lesions (35.33\%), 17 small HCC lesions (44.74\%), and 7 large HCC lesions $(100.00 \%)$. In the US examination, there were 55 micro HCC lesions (36.67\%), 18 small HCC lesions (47.37\%), and 7 large HCC lesions (100.00\%). In the MRI examination, there were 78 micro HCC lesions (52.00\%), 19 small HCC lesions (50.00\%), and 7 large HCC lesions (100.00\%). In the 
Table VIII. Comparison of detection rates of CT, US and MRI in the two groups [n (\%)].

\begin{tabular}{lcccc}
\hline Variables & CT & US & MRI & US combined with MRI \\
\hline True positive [n (\%)] & $75(22.80)$ & $77(23.40)$ & $100(30.40)$ & $79(24.01)$ \\
False positive [n (\%)] & $99(30.09)$ & $96(29.18)$ & $71(21.58)$ & $93(28.27)$ \\
True negative [n (\%)] & $70(21.28)$ & $73(22.19)$ & $98(29.79)$ & $76(23.10)$ \\
False negative [n (\%)] & $85(25.83)$ & $83(25.23)$ & $60(18.24)$ & $81(24.62)$ \\
Sensitivity (\%) & $46.88^{\mathrm{a}}$ & $48.13^{\mathrm{a}}$ & 62.50 & $49.38^{\mathrm{a}}$ \\
Specificity (\%) & $41.42^{\mathrm{a}}$ & $43.20^{\mathrm{a}}$ & 57.99 & $44.97^{\mathrm{a}}$ \\
Positive predictive value (\%) & $43.10^{\mathrm{a}}$ & $44.51^{\mathrm{a}}$ & 58.48 & $45.93^{\mathrm{a}}$ \\
Negative predictive value (\%) & $45.16^{\mathrm{a}}$ & $46.79^{\mathrm{a}}$ & 62.03 & $48.40^{\mathrm{a}}$ \\
\hline
\end{tabular}

${ }^{\mathrm{a}}<0.05$, compared with MRI in the same group. CT, computed tomography; US, ultrasound; MRI, magnetic resonance imaging.

US combined with MRI detection, there were 65 micro HCC lesions (43.33\%), 26 small HCC lesions (68.42\%), and 7 large HCC lesions (100.00\%). Since the diagnostic efficiency of $\mathrm{CT}$ is lower than the other three methods, it was used as a normal control. When the lesion type of RHCC patients was small $\mathrm{HCC}$, there were no significant differences in the coincidence rate among CT, US, MRI, and US combined with MRI ( $\mathrm{P}>0.05)$. When the lesion type was large HCC, there were no significant differences in the coincidence rate among US, MRI, and US combined with MRI $(P>0.05)$. When the lesion type was micro HCC, the coincidence rate of MRI alone was significantly higher than that of US $(\mathrm{P}<0.05)$. There was no significant difference in the coincidence rate between US alone and US combined with MRI $(\mathrm{P}>0.05)$, as shown in Table VII.

Comparison of CT, US and MRI detection rate. PHCC was assumed as true positive, and RHCC was assumed as true negative. Two groups were combined as one group. The sensitivity, specificity, positive predictive value and negative predictive value of MRI were significantly better than those of the other three methods, and the differences were statistically significant $(\mathrm{P}<0.05)$. The other three methods had no significantly different results (Table VIII).

\section{Discussion}

PHCC is a malignant tumor that is common in middle-aged males (17). At present, US is mainly used for early screening and diagnosis of HCC. However, there is a variety of factors that may affect the results of US examination, such as the weight and body position of the patient, as well as the instrument performance. In addition, some HCCs do not conform to the 'fast in and fast out' of US indicator. Thus, it is difficult to identify HCC by using US alone (18). MRI provides high resolution and is applied to complement US in the detection of lesions (19). Although RHCC is derived from PHCC, its pathological and imaging features have been altered after the treatment and the natural development of HCC cells $(20,21)$. This may be the reason why the lesion size is smaller than in PHCC. This study compared the value of US combined with MRI in the diagnostic process of PHCC and RHCC, to determine a more accurate and effective imaging technique to diagnose, screen and identify PHCC, as well as to improve the detection rate of RHCC.

Although there was no difference in age, sex and other general clinical data between the two groups of patients, the size of lesions in the PHCC group was significantly larger than that in the RHCC group. This is consistent with the findings of Escartin et al (22). In the comparison of the two groups, we found that the coincidence rates of US, MRI, and US combined with MRI in the PHCC group were all significantly higher than those in the RHCC group. This may be because the lesion of RHCC is smaller than that of PHCC, and this makes it more difficult to detect. The coincidence rate of using MRI alone was higher than that of using US alone and higher than using US combined with MRI. This suggests that the detection rate of RHCC is higher when MRI is used alone. In terms of PHCC group, the coincidence rate of using MRI alone was significantly higher than that of using US alone or using US combined MRI. The reason of the poor result of the combined detection may be that although US combined with MRI can complement each other, it may also lead to a decrease in the correct rate due to conflict between MRI diagnosis and US misdiagnosis. There was no significant difference between the use of US alone and US combined with MRI. Furthermore, through comparing and analyzing the performances of US, MRI, and US combined with MRI in detecting different RHCC lesions, we found that when the lesions were of micro HCC type, the coincidence rate of MRI alone was significantly higher than that of US alone $(\mathrm{P}<0.05)$. There was no significant difference in the coincidence rate between US alone and US combined with MRI ( $P>0.05)$.Xiao et al (23) reported that in the case of lesions with a diameter of $\leq 2 \mathrm{~cm}$, the isoechoic enhancement rates of RHCC in arterial phase were significantly higher than those of PHCC. RHCC is more likely to be diagnosed as 'fast in and slow out'. Shagdarsuren et al (24) conducted a study regarding the role of US in the diagnosis of RHCC. It was reported that the size and location of the lesion, and the thickness of the abdominal wall are independent factors that prevent the US angiography from being evaluated. This is also demonstrated in the results of the present study: In the case of lesions with a diameter of $\leq 2 \mathrm{~cm}$, the coincidence rate 
of US was only $36.67 \%$, which is far less than the coincidence rate of lesions with a diameter of $>2 \mathrm{~cm}$. Therefore, apart from the correct choice of the imaging technique, tumor markers, such as AFP should be taken into consideration for the comprehensive diagnosis of RHCC (25).

In summary, the diagnostic efficacy of MRI for PHCC was superior to that of CT, US, and US combined with MRI. There was no significant difference among CT, US, and US combined with MRI. The diagnostic efficacy of MRI for RHCC was higher than that of CT, and US was higher than US combined with MRI. For the detection of micro HCC lesions, the diagnostic efficacy of MRI alone was the highest. MRI, US, CT, and MRI combined with US had better performance in the diagnosis of PHCC than in the diagnosis of RHCC. The diagnosis of RHCC should be combined with other indicators to ensure a comprehensive identification.

\section{Acknowledgements}

Not applicable.

\section{Funding}

No funding was received.

\section{Availability of data and materials}

The datasets used and/or analyzed during the present study are available from the corresponding author on reasonable request.

\section{Authors' contributions}

$\mathrm{XJ}$ wrote the manuscript and was involved in the conception of the study. FL and PY analyzed and interpreted the patient data. SZ and YL were responsible for the US and MRI result analysis. HL assisted with statistical analysis. All authors read and approved the final manuscript.

\section{Ethics approval and consent to participate}

The study was approved by the Ethics Committee of Qingdao Women and Children's Hospital (Qingdao, China). The patients and their families were informed, and informed consents were signed. All patients who participated in this research had complete clinical data.

\section{Patient consent for publication}

Not applicable.

\section{Competing interests}

The authors declare that they have no competing interests.

\section{References}

1. Hu Z, Zhou J, Xu X, Li Z, Zhou L, Wu J, Zhang M and Zheng S: Salvage liver transplantation is a reasonable option for selected patients who have recurrent hepatocellular carcinoma after liver resection. PLoS One 7: e36587, 2012.
2. Yang YY, Wang SJ, Yang LY, Lirng JF, Huang CC, Liang JF, Lee FY, Hwang SJ, Huang CC and Kirby R: Effects of a new parallel primary healthcare centre and on-campus training programme on history taking, physical examination skills and medical students' preparedness: A prospective comparative study in Taiwan. BMJ Open 7: e016294, 2017.

3. Cong WM, Bu H, Chen J, Dong H, Zhu YY, Feng LH, Chen J and Committee G; Guideline Committee: Practice guidelines for the pathological diagnosis of primary liver cancer: 2015 update. World J Gastroenterol 22: 9279-9287, 2016.

4. Rojas A, Zhang P, Wang Y, Foo WC, Muñoz NM, Xiao L, Wang J, Gores GJ, Hung MC and Blechacz B: A positive TGF- $\beta / \mathrm{c}-\mathrm{KIT}$ feedback loop drives tumor progression in advanced primary liver cancer. Neoplasia 18: 371-386, 2016.

5. Onitilo AA, Engel JM, Greenlee RT and Mukesh BN: Breast cancer subtypes based on ER/PR and Her2 expression: Comparison of clinicopathologic features and survival. Clin Med Res 7: 4-13, 2009.

6. Rubin J, Ayoub N, Kaldas F and Saab S: Management of recurrent hepatocellular carcinoma in liver transplant recipients: A systematic review. Exp Clin Transplant 10: 531-543, 2012.

7. Oliva MR and Saini S: Liver cancer imaging: role of CT, MRI, US and PET. Cancer Imaging 4: S42-S46, 2004.

8. Chen Q, Shang W, Zeng C, Wang K, Liang X, Chi C, Liang X, Yang J, Fang C and Tian J: Theranostic imaging of liver cancer using targeted optical/MRI dual-modal probes. Oncotarget 8: 32741-32751, 2017.

9. Snowberger N, Chinnakotla S, Lepe RM, Peattie J, Goldstein R, Klintmalm GB and Davis GL: Alpha fetoprotein, ultrasound, computerized tomography and magnetic resonance imaging for detection of hepatocellular carcinoma in patients with advanced cirrhosis. Aliment Pharmacol Ther 26: 1187-1194, 2007.

10. Dumitrescu CI, Gheonea IA, Săndulescu L, Surlin V, Săftoiu A and Dumitrescu D: Contrast enhanced ultrasound and magnetic resonance imaging in hepatocellular carcinoma diagnosis. Med Ultrason 15: 261-267, 2013.

11. Sun X, Li RU, Zhang B, Yang Y and Cui Z: Treatment of liver cancer of middle and advanced stages using ultrasound-guided percutaneous ethanol injection combined with radiofrequency ablation: A clinical analysis. Oncol Lett 11: 2096-2100, 2016.

12. Shi J, Liu TW, Chen J, Green D, Jaffray D, Wilson BC, Wang F and Zheng G: Transforming a targeted porphyrin theranostic agent into a PET imaging probe for cancer. Theranostics 1: 363-370, 2011.

13. Huang L, Li GM, Zhu JY, Li Z, Li T and Leng XS: Efficacy of sorafenib after liver transplantation in patients with primary hepatic carcinoma exceeding the Milan criteria: A preliminary study. OncoTargets Ther 5: 457-462, 2012.

14. Malagari K, Pomoni M, Sotirchos VS, Moschouris H, Bouma E, Charokopakis A, Kelekis AD, Koundouras D, Filippiadis D, Chatziioannou A, et al: Long term recurrence analysis post drug eluting bead (deb) chemoembolization for hepatocellular carcinoma (hcc). Hepatogastroenterology 60: 1413-1419, 2013.

15. Lee CH, Brubaker LM, Gerber DA, Ku YM, Kim YH, Shin SS and Semelka RC: MRI findings of recurrent hepatocellular carcinoma after liver transplantation: Preliminary results. J Magn Reson Imaging 33: 1399-1405, 2011.

16. Le Moigne F, Durieux M, Bancel B, Boublay N, Boussel L, Ducerf C, Berthezène Y and Rode A: Impact of diffusion-weighted MR imaging on the characterization of small hepatocellular carcinoma in the cirrhotic liver. Magn Reson Imaging 30: 656-665, 2012.

17. Bruix J, Reig M and Sherman M: Evidence-based diagnosis, staging, and treatment of patients with hepatocellular carcinoma. Gastroenterology 150: 835-853, 2016.

18. Hu Z, Lv G, Li Y, Li E, Li H, Zhou Q, Yang B and Cao W: Enhancement of anti-tumor effects of 5-fluorouracil on hepatocellular carcinoma by low-intensity ultrasound. J Exp Clin Cancer Res 35: 71, 2016.

19. Furlan A, Marin D, Cabassa P, Taibbi A, Brunelli E, Agnello F, Lagalla R and Brancatelli G: Enhancement pattern of small hepatocellular carcinoma (HCC) at contrast-enhanced US (CEUS), MDCT, and MRI: Intermodality agreement and comparison of diagnostic sensitivity between 2005 and 2010 American Association for the Study of Liver Diseases (AASLD) guidelines. Eur J Radiol 81: 2099-2105, 2012.

20. Wakayama K, Kamiyama T, Yokoo H, Orimo T, Shimada S, Einama T, Kamachi H and Taketomi A: Huge hepatocellular carcinoma greater than $10 \mathrm{~cm}$ in diameter worsens prognosis by causing distant recurrence after curative resection. J Surg Oncol 115: 324-329, 2017. 
21. Yang X, Wang D, Ma Y, Zhao Q, Fallon JK, Liu D, Xu XE, Wang Y, He Z and Liu F: Theranostic nanoemulsions: Codelivery of hydrophobic drug and hydrophilic imaging probe for cancer therapy and imaging. Nanomedicine (Lond) 9: 2773-2785, 2014.

22. Escartin A, Sapisochin G, Bilbao I, Vilallonga R, Bueno J, Castells L, Dopazo C, Castro E, Caralt M and Balsells J: Recurrence of hepatocellular carcinoma after liver transplantation. Transplant Proc 39: 2308-2310, 2007.

23. Xiao JD, Zhu WH and Shen SR: Evaluation of hepatocellular carcinoma using contrast-enhanced ultrasonography: Correlation with microvessel morphology. Hepatobiliary Pancreat Dis Int 9: 605-610, 2010.
24. Shagdarsuren B, Tamai H, Shingaki N, Mori Y, Maeshima S, Nuta J, Maeda Y, Moribata K, Niwa T, Deguchi H, et al: Contribution of contrast-enhanced sonography with perfluorobutane microbubbles for diagnosis of recurrent hepatocellular carcinoma. J Ultrasound Med 35: 1383-1391, 2016.

25. Nörthen A, Asendorf T, Walson PD and Oellerich M: Diagnostic value of alpha-1-fetoprotein (AFP) as a biomarker for hepatocellular carcinoma recurrence after liver transplantation. Clin Biochem 52: 20-25, 2018.

(i) (9) This work is licensed under a Creative Commons EY NG ND Attribution-NonCommercial-NoDerivatives 4.0 International (CC BY-NC-ND 4.0) License. 